\title{
Les lipides : une matière première alternative pour la synthèse de polymères de spécialités
}

\author{
Guillaume CHOLLET ${ }^{1}$ \\ Benoit GADENNE ${ }^{1}$ \\ Carine ALFOS ${ }^{1}$ \\ Henri CRAMAIL ${ }^{2}$ \\ 1 ITERG, \\ Institut des Corps Gras, \\ 11 rue Gaspard Monge, \\ 33600 Pessac, \\ France (www.iterg.com) \\ $<$ c.alfos@iterg.com > \\ ${ }^{2}$ Laboratoire de Chimie des Polymères \\ Organiques, \\ UMR 5629 CNRS-Université Bordeaux1- \\ IPB, \\ 16, Avenue Pey-Berland, \\ 33607 Pessac Cedex, \\ France (www.lcpo.fr)
}

À l'heure actuelle, la dépendance de I'industrie chimique aux ressources fossiles reste très importante (95\% des produits chimiques sont d'origine fossile) et constitue, à terme, des risques économiques (augmentation des coûts), écologiques (augmentation de l'émission des gaz à effets de serre) et politiques. De plus, la raréfaction de ces ressources non renouvelables fait partie, avec le changement climatique, la pollution, l'augmentation de la population, les ressources limitées en eau et l'érosion de la biodiversité, d'un ensemble de problématiques qui constituent l'enjeu même du développement durable. L'utilisation des ressources renouvelables s'inscrivant aussi dans cette démarche de développement durable, apparaît comme une alternative, voire une priorité, afin de répondre au mieux aux problématiques de durabilité (Rapport PIPAME, 2010).

\begin{abstract}
Currently, the dependence of the chemical industry on fossil fuels is very important (95\% of chemicals from fossil fuels). In addition, the scarcity of nonrenewable resources is one - with climate change, pollution, increasing population, limited water resources and biodiversity erosion - of the sustainable development challenges. The use of renewable resources appears as an alternative or even a priority in order to solve the challenges of sustainability. The French chemical industry is a very active area with a GDP of around 18 billion euros and 1.3 billion euros of investment in research and development. However, the sector also faces a number of difficulties due to the decreased activity of its petrochemical industries, the phenomena of offshoring and concentrations. Indeed, the development of new bio-based materials is a major challenge and vegetable oils can be a source of such bio-based materials.
\end{abstract}

Key words: lipid, polyols, polyurethanes, bio-based polymers

L'industrie chimique française est un secteur très actif avec un PIB d'environ 18 milliards d'euros et 1,3 milliard d'euros d'investissements en recherche et développement. Cependant ce secteur doit aussi faire face à un certain nombre de difficultés dues, entre autres, à la diminution de l'activité pétrochimique et de ses filières et aux phénomènes de délocalisations et de concentrations. Le développement de nouveaux matériaux biosourcés constitue donc un enjeu majeur pour l'industrie chimique en France. En 2010, la capacité annuelle mondiale de production de "résines" bio-sourcées était de 0,72 million de tonnes, à comparer aux 265 millions de tonnes pour la production mondiale de " matériaux plastiques ", ce qui montre à la fois la part très faible que représente actuellement la chimie du végétal, mais aussi l'ampleur des marchés potentiels.

Les huiles végétales sont, sur le plan industriel, d'une importance significative et sont couramment utilisées pour de nombreuses applications, parmi lesquelles la synthèse de polymères biosourcés. Les huiles de palme, de soja et de colza représentent les trois quarts de la production mondiale $d$ 'huiles végétales (figure 1). Elles sont considérées comme I'une des sources de matières renouvelables les plus importantes du fait de leur faible coût, de leur abondance et des nombreuses possibilités de transformations chimiques qu'elles offrent. La production $d$ 'huiles végétales est destinée principalement au secteur alimentaire. La transformation de ces huiles par l'industrie représente $24 \%$ du marché mondial (figure 2).

\section{Matières premières biosourcées}

L'amidon, la cellulose, la lignine, les sucres et les huiles végétales constituent les principales matières premières biosourcées et sont déjà présentes dans les secteurs de l'énergie, de l'industrie, de

Pour citer cet article : Chollet $\mathrm{G}$, Gadenne $\mathrm{B}$, Alfos $\mathrm{C}$, Cramail $\mathrm{H}$. Les lipides : une matière première alternative pour la synthèse de polymères de spécialités. OCL $2012 ; 19(1)$ : 39-50. doi : 10.1684/ocl.2012.0428 


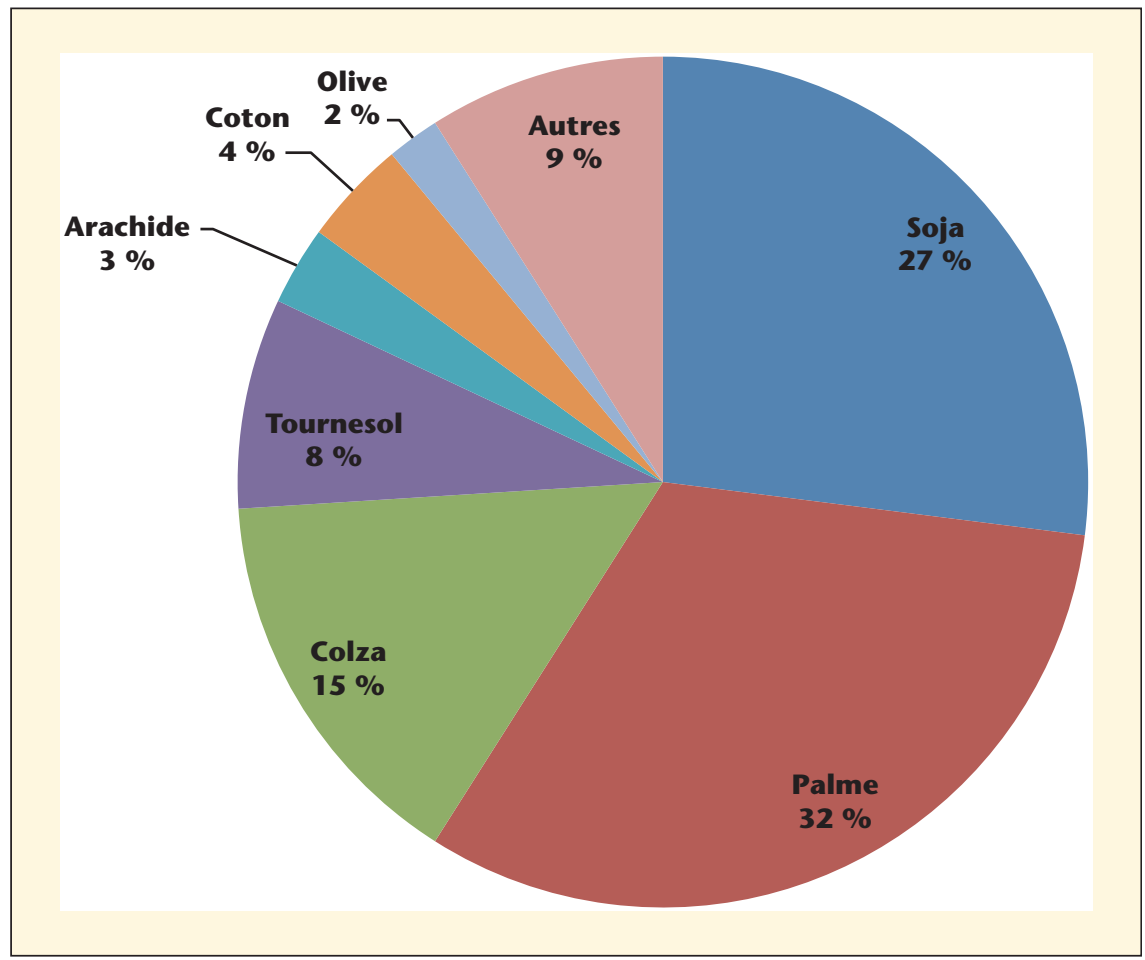

Figure 1. Production mondiale d'huiles végétales en 2008.

la chimie fine, de la pharmacie, des cosmétiques et dans I'alimentaire.

\section{Les polysaccharides}

1) L'amidon se trouve dans de nombreuses ressources végétales telles que la pomme de terre, le blé, le maïs, le manioc, etc. L'amidon est un polysaccharide composé de l'enchaînement covalent d'unités D-glucose. Son hydrolyse acide complète libère de 98 à $99 \%$ de D-glucose. II est constitué de deux homopolymères, I'amylose, macro-

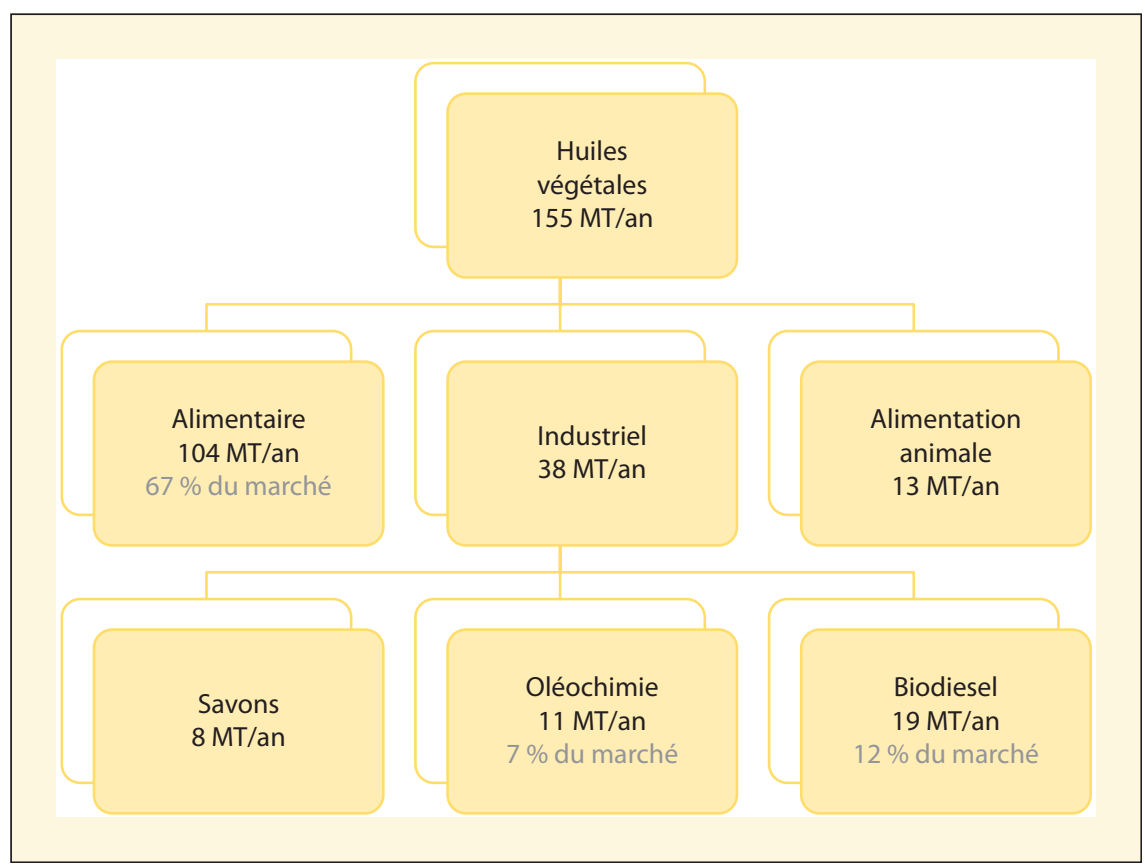

Figure 2. Production mondiale et débouchés des huiles végétales.

molécule essentiellement linéaire et I'amylopectine, macromolécule ramifiée. L'amylose constitue 20 à $30 \%$ de l'amidon et I'amylopectine 70 à $80 \%$. La fraction non glucidique représente, quant à elle, 1 à $2 \%$ de la masse totale des amidons (figure 3).

La structure chimique de l'amidon lui confère des propriétés intéressantes en termes de réactivité. L'amidon peut subir un certain nombre de modifications chimiques au niveau des fonctions alcool primaires et secondaires des unités glycosyle par le biais des réactions d'oxydation, d'estérification, d'éthérification, et au niveau de la liaison glycosidique et de la fonction pseudo-aldéhydique avec des réactions d'hydrogénation. Le procédé d'extrusion réactive est parfaitement adapté à ce type de transformations. L'amidon peut également subir des modifications physiques, physico-chimiques (dextrinisation) ou biologiques (hydrolyse).

Les capacités de production en 2010 de résines à base $d^{\prime}$ amidon étaient de $288 \mathrm{ktpa}^{1}$ et représentaient pas moins de $40 \%$ des capacités mondiales de production des résines biosourcées.

Les principaux producteurs de résines à base d'amidon se situent en Europe avec Novamont (80 ktpa), Biotec (50 ktpa), Rodenburg (40 ktpa), Biop (20 ktpa) et Bioresins (20 ktpa). On peut également citer Cereplast aux États-Unis avec 22 ktpa et Earthsoul India en Inde avec 20 ktpa (Rapport ADEME 2011) (figure 4).

2) La cellulose est le polysaccharide le plus abondant sur terre. II se trouve principalement dans le bois (50\%) mais également dans le coton (97\%). Des sources alternatives comme les algues, les membranes de champignons et de bactéries sont en cours de développement.

La structure de la cellulose consiste en I'enchaînement covalent d'unités de répétition de type cellobiose, les unités élémentaires glucosidiques étant liées entre elles par des liaisons $\beta-(1 \rightarrow 4)$ (figure 5).

La formation de nombreuses liaisons hydrogène intra- et intermoléculaires entre les groupements hydroxyle confère une forte cristallinité à la

\footnotetext{
${ }^{1} \mathrm{KT} / \mathrm{an}$
} 

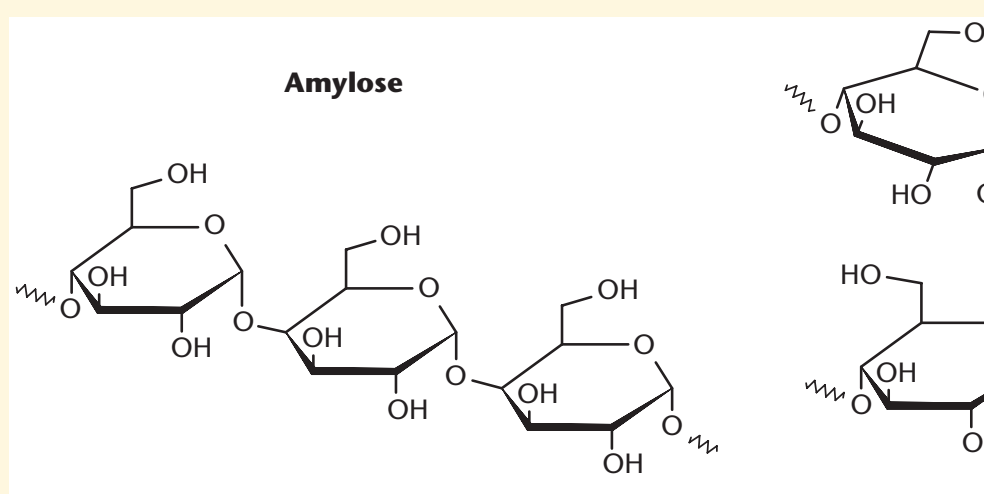

$\mathrm{OH}$

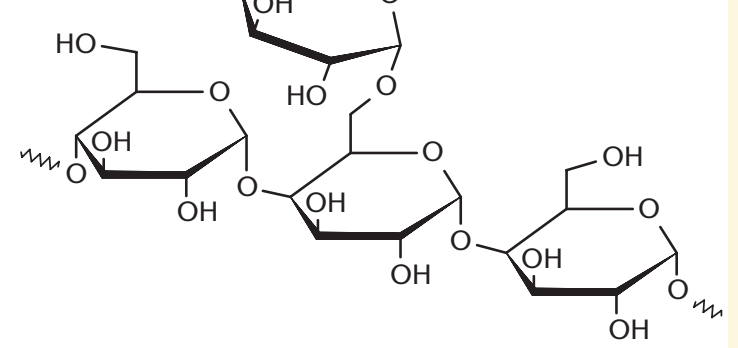

Figure 3. Structure chimique de l'amidon.

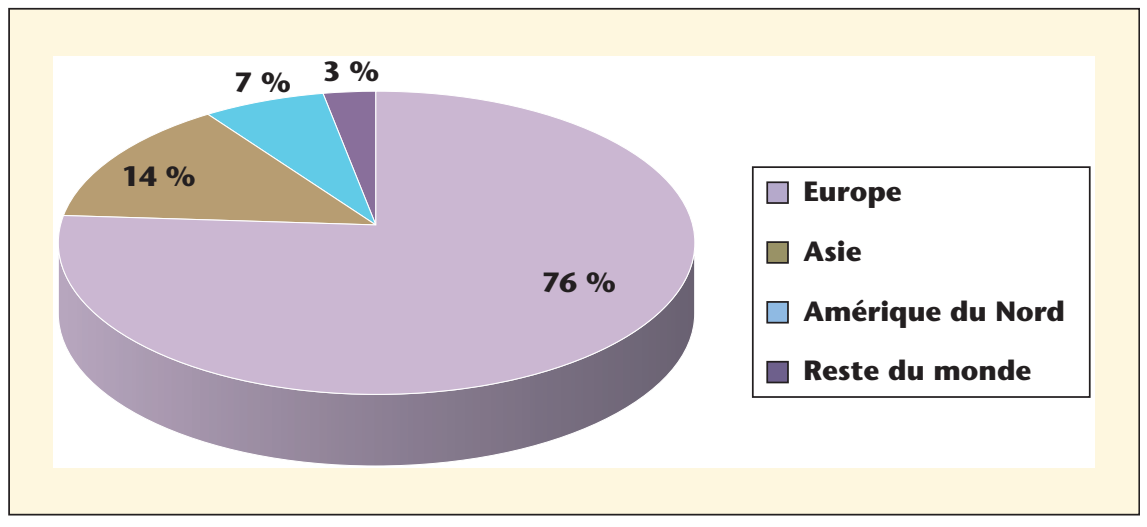

Figure 4. Répartition géographique de la production de résines à base d'amidon en 2010.

cellulose entraînant son insolubilité dans l'eau.

Les groupements hydroxyle de la cellulose peuvent réagir partiellement ou totalement avec différents réactifs chimiques pour donner des dérivés cellulosiques possédant des propriétés propres. Les modifications chimiques peuvent être des estérifications (acétate de cellulose) ou des éthérifications (hydroxyéthyl cellulose).

Les résines à base cellulosique ne représentent que $3 \%$ des capacités de résines biosourcées, ce qui est notamment dû aux procédés de transformations dont les coûts s'avèrent trop

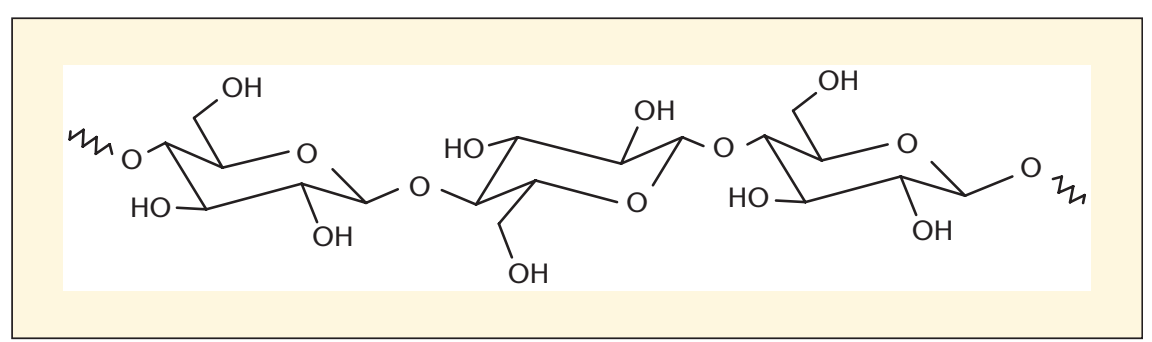

Figure 5. Structure chimique de la cellulose. élevés. Les marchés ciblés par ces résines sont ceux des emballages cosmétiques et agroalimentaires.

Ces résines sont essentiellement produites en Allemagne par les sociétés Fkur et Tecnaro, et aux États-Unis par les sociétés Rotuba et Eastman. Ce dernier est même le premier producteur de résines à base de cellulose avec 15 ktpa (Rapport ADEME 2011).

3) La transformation, impliquant la déconstruction et la dépolymérisation de la biomasse par le biais de traitements hydrolytique et/ou enzymatique, reste aujourd'hui un des enjeux majeurs en chimie durable pour la production de composés chimiques, la "plateforme sucre »-qualifiée d'or vert ! - étant une source inépuisable d'intermédiaires chimiques et notamment de synthons monomères tel l'acide lactique. Le poly (acide lactique) (PLA), de la famille des polyesters, est aujourd'hui un des principaux polymères biosourcés développés industriellement. II est majoritairement obtenu par polymérisation par ouverture de cycle du lactide, dérivé de l'acide lactique (figure 6).

La production d'acide lactique peut être réalisée à partir du maïs (société Natureworks), de la canne à sucre, de la betterave, du manioc (société Purac). Le groupe néerlandais Purac est le leader mondial de la production d'acide lactique avec 160 ktpa.

Natureworks est le premier producteur mondial de résines à base de PLA avec une production représentant $95 \%$ des 


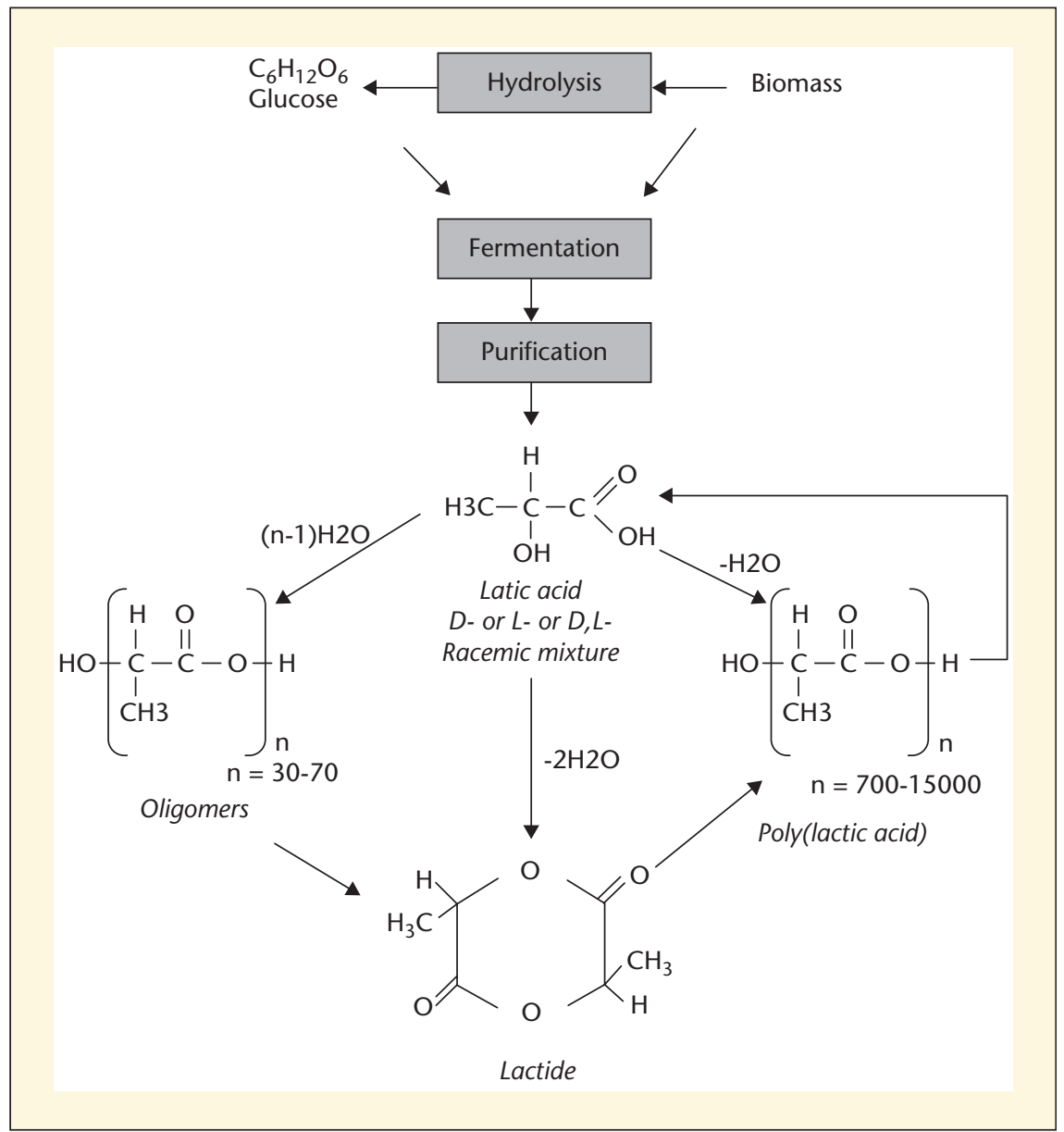

Figure 6. Synthèse du PLA. capacités mondiales, les $5 \%$ restants étant produits par le groupe chinois Zhejiang Hisun.

Les matières premières sources $d$ 'hexoses, utilisées pour la production de poly (hydroxyalcanoate)s (PHA), selon des procédés de biotechnologies qualifiées de "blanches", sont très nombreuses. En effet, outre la canne à sucre, la betterave, les huiles végétales et le maïs, les déchets alimentaires, les eaux usées, la sève d'érable peuvent aussi être avantageusement utilisés.

La production mondiale de résines à base de PHA est estimée à 43 ktpa en 2010 et la société brésilienne Biocycle est pratiquement en position de monopole puisque ses capacités de production sont

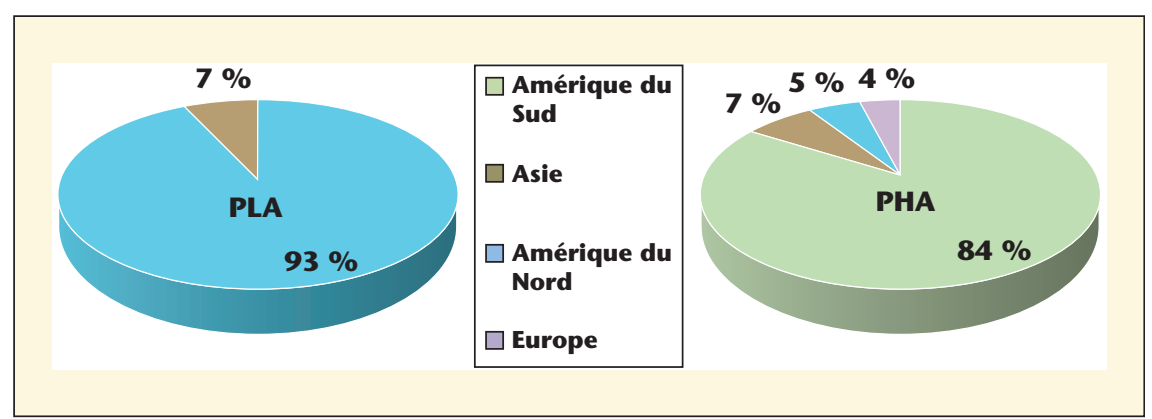

Figure 7. Répartition géographique de la production de résines à base PLA et PHA en 2010. de 36 ktpa (Rapport ADEME

2011) (figure 7).

Les polysaccharides sont également une source majeure de polyols. Ces derniers représentent une classe importante d'intermédiaires réactionnels (synthons monomères) en chimie des polymères, notamment pour la synthèse des polyuréthanes et des polyesters.

Le Cerenol ${ }^{\mathrm{TM}}$, commercialisé par Dupont de Nemours, est un polyol issu du maïs et possède un taux de carbone renouvelable de $100 \%$. II est utilisé pour la synthèse de fibres polyuréthanes. Le Néosorb ${ }^{\circledR}$ et le Polysorb ${ }^{\circledR}$ sont deux polyols commercialisés par Roquette. Le premier est à base de sorbitol, polyol obtenu par hydrogénation du glucose et le second, à base d'isosorbide, obtenu par déshydratation du sorbitol. L'isosorbide peut être utilisé pour la synthèse de polymères de spécialité.

\section{Les huiles végétales}

Les huiles végétales peuvent également être sources de nouveaux polyols en remplacement des polyols d'origine pétrochimique. En effet, la structure même des huiles (longueur de chaînes, degré d'insaturation, stéréochimie des doubles liaisons) permet d'envisager la réalisation de composés multiples et variés. Une huile est un mélange de triglycérides (triesters) formés par condensation d'acides gras et du glycérol. Le nombre élevé de types d'acides gras (jusqu'à 24) potentiellement présents dans chaque corps gras et les multiples combinaisons possibles avec le glycérol font que les corps gras sont des mélanges plus ou moins complexes de composés à l'origine de propriétés variables d'une huile à l'autre, chacune contenant donc des triglycérides de nature différente (figure 8).

Des études académiques décrivent I'utilisation des huiles végétales et de leurs dérivés (triglycérides et esters méthyliques d'acides gras) en tant que matières premières pour la préparation d'agro-matériaux aux propriétés spécifiques dans une multitude d'applications (Güner et al., 2006 ; Sharma et al., 2006). La plupart des huiles, hormis I'huile de ricin qui possède déjà des groupements hydroxyle natifs, peuvent donner des polyols après transformations chimiques. Des études décrivent des modifications oléochimiques des huiles insaturées (principalement composées d'acide oléique 


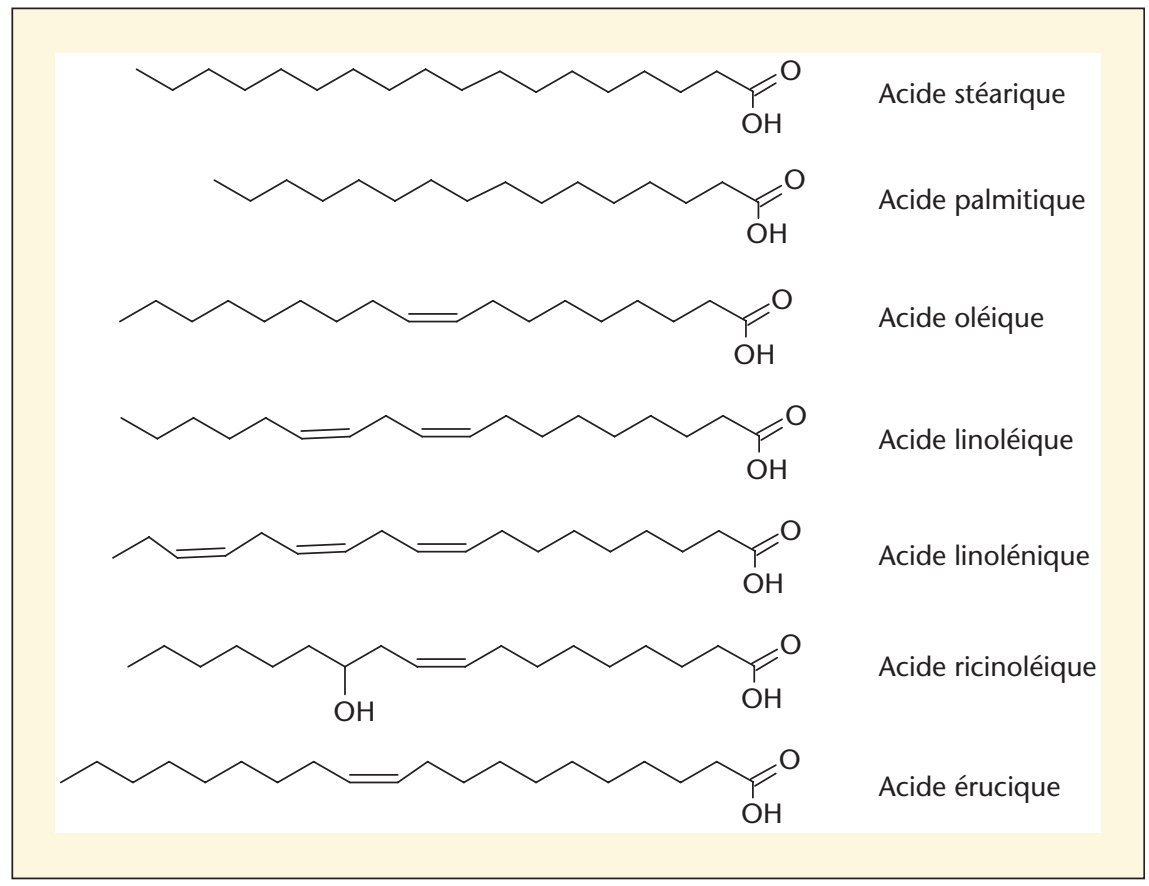

Figure 8. Exemples d'acides gras issus d'huiles végétales.

et linoléique) pour produire des polyols qui peuvent être utilisés en tant que monomères fonctionnels pour la synthèse de polymères biodégradables (Galia et al., 2010).

Les fonctions ester du triglycéride peuvent subir une réaction de transestérifica-

tion (figure 9) en présence d'un alcool tel que le méthanol, l'éthanol ou le glycérol et d'un catalyseur homogène ou hétérogène (Meher et al., 2006). Les catalyseurs les plus communément utilisés sont le méthanolate de sodium, I'hydroxyde de sodium ou de potassium. Les catalyseurs acides sont plus particulièrement

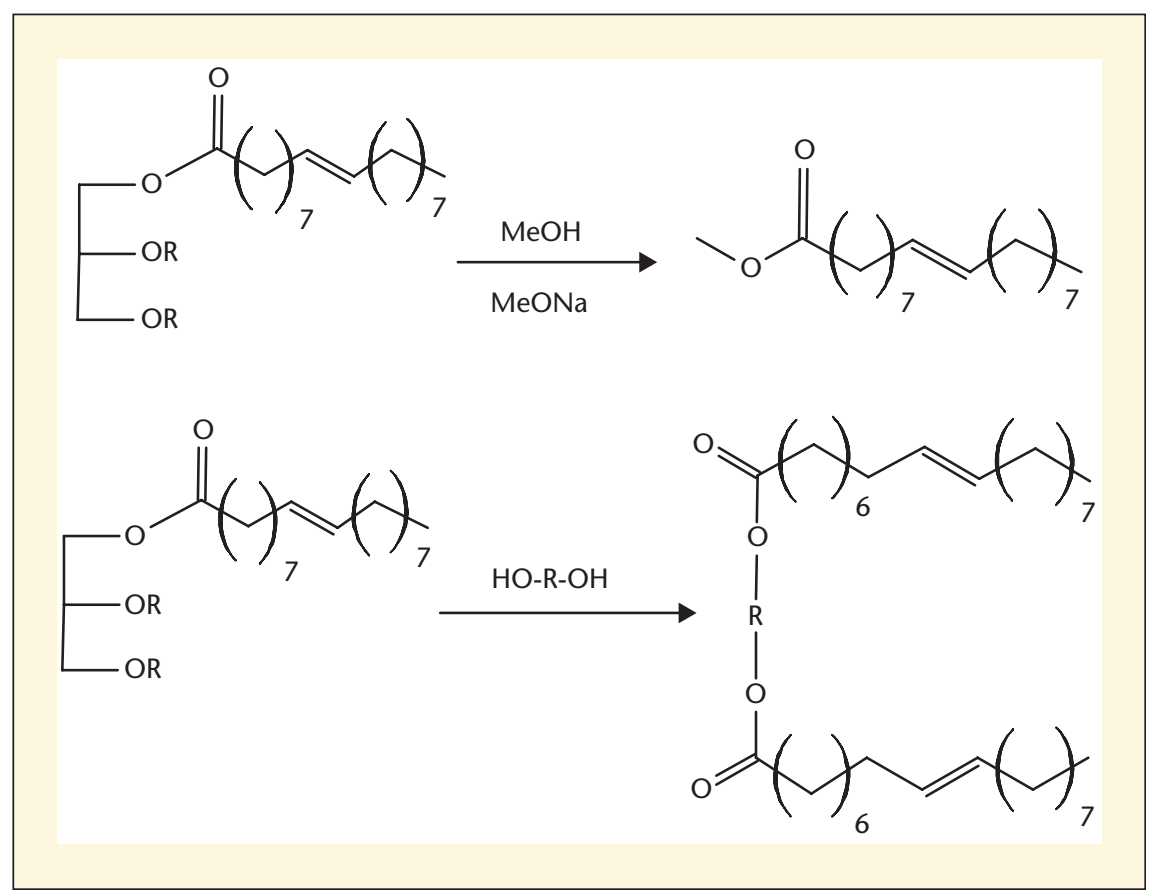

Figure 9. Réactions de transestérification. employés dans le cas de la transestérification d'huiles ayant une quantité importante d'acides gras libres et d'eau (Nakatani et al., 2009).

Les réactions d'époxydation (figure 10) sur des esters d'acides gras ou sur les triglycérides sont classiquement utilisées et conduisent, après ouverture des cycles oxirane par différents nucléophiles (alcools, amines), à des polyols contenant des alcools secondaires. Les réactifs utilisés pour la réaction d'époxydation sont des peracides comme l'acide métachloroperbenzoïque (m-CPBA) (De Torres et al., 2011) ou le peracide monoperoxyphthalate hexahydrate de magnésium (MMPP). II est également possible de former le peracide in-situ en mélangeant de l'eau oxygénée et de I'acide acétique (ou formique) (Zlatanic et al., 2002).

Des fonctions alcool primaires peuvent aussi être introduites par réactions d'hydroformylation (figure 11) ou d'ozonolyse (figure 12) sur des triglycérides suivies de la réduction des fonctions aldéhyde ainsi obtenues (Petrovic et al., 2000 ; Zlatanic et al., 2004 ; Petrovic et al., 2005 ; Zlatanic et al., 2009 ; Petrovic et al., 2010). Les réactifs utilisés lors de l'étape de réduction sont le borohydrure de sodium (Petrovic et al., 2005) ou le dihydrogène en présence de nickel de Raney (Narine et al., 2007).

La fonctionnalisation des doubles liaisons des acides gras peut également se faire via la réaction radicalaire de type thiol-ène (figure 13). Les radicaux peuvent être générés par action de la lumière ou par décomposition thermique d'un composé azoïque tel I'AIBN. Dans le cas où le thiol utilisé possède également une fonction alcool (mercaptoéthanol par exemple), les polyols ainsi formés présentent exclusivement des fonctions alcool primaires (Bantchev et al., 2009).

Un certain nombre de polyols commerciaux issus d'huile végétale existent. Ils sont principalement utilisés dans la synthèse de polyuréthanes. Citons ceux issus d'huile de soja comme Renuva ${ }^{\mathrm{TM}}$ commercialisé par Dow Chemical, $\mathrm{BiOH}^{\mathbb{R}}$ de la société Cargill, $\left.A g r o\right|^{\mathbb{R}}$ de BioBased Technologies, Jeffadd ${ }^{\mathrm{TM}}$ de la société Huntsman et Soyol ${ }^{\mathbb{R}}$ de Urethane Soy Systems ou encore Luprano ${ }^{\mathbb{R}}$ de BASF, issu de l'huile de ricin et la gamme Radia ${ }^{\mathbb{R}}$ d'Oléon à partir des huiles de tournesol et de colza. 


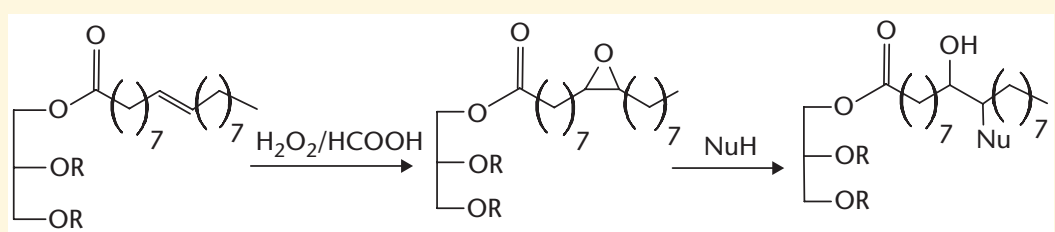

avec $\mathrm{NuH}=$ alcool ou amine

Figure 10. Réaction d'époxydation et d'ouverture de cycle.

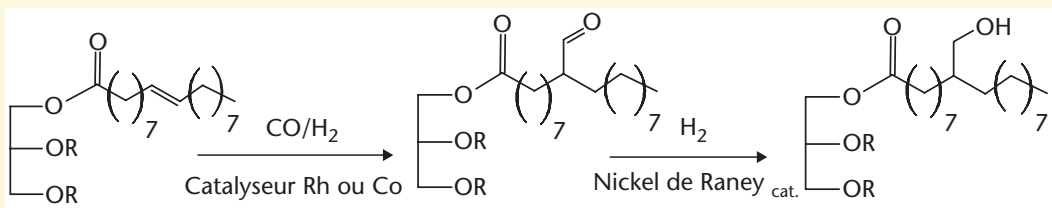

Figure 11. Réaction d'hydroformylation et de réduction.

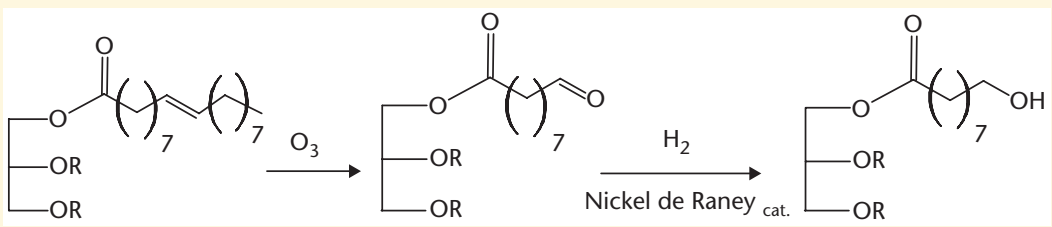

Figure 12. Réaction d'ozonolyse et de réduction.

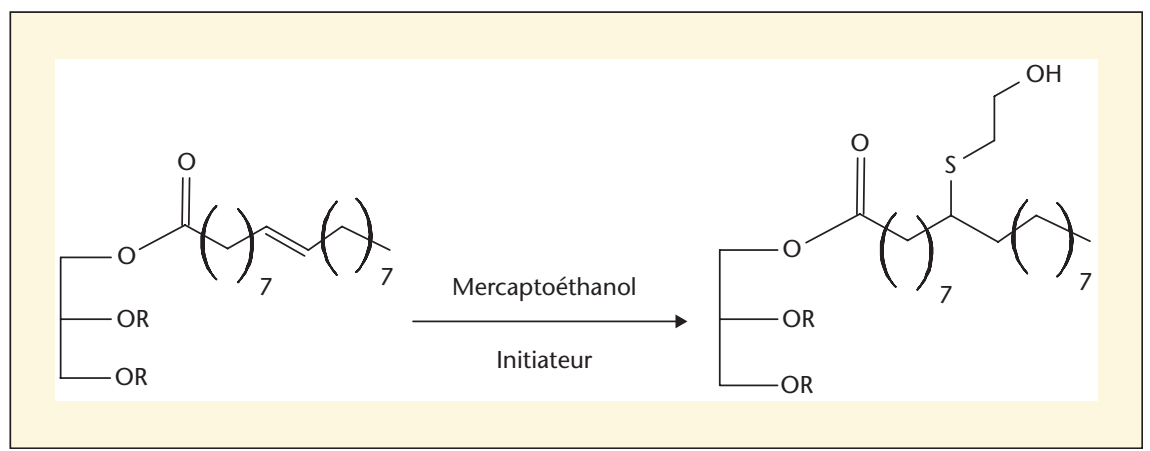

Figure 13. Réaction thiol-ène sur les insaturations des acides gras.

\section{Travaux de l'unité mixte technologique (UMT) Polygreen}

Dans l'optique de valorisation de la biomasse, le Centre technique industriel en oléochimie, ITERG, et le Laboratoire de chimie des polymères organiques (LCPO-Université de Bordeaux) ont additionné leurs "savoir-faire " en créant une Unité mixte technologique (UMT Polygreen labellisée en 2007 par le ministère de l'Agriculture et de la Pêche). L'objectif de l'UMT est de mettre au point de nouveaux polymères biosourcés à partir de monomères issus de la transformation oléochimique des huiles végétales (éventuellement couplés avec des dérivés issus d'autres filières) et utilisant des procédés de synthèses éco-compatibles. Les objectifs sont ainsi de concevoir de nouveaux "synthons monomères verts " de structure chimique et de fonctionnalité bien définies (Palaskar et al., 2010 ; Boyer et al., 2010 ; Palaskar et al., 2012 ; Foltran et al., 2012), d'optimiser à grande échelle leurs procédés de synthèse (phase scale-up) et d'utiliser ces monomères pour la synthèse de polyuréthanes et polyesters biosourcés originaux.

Dans le cadre de cette collaboration, les actions de recherche ont été principalement orientées vers la modification chimique d'esters méthyliques d'acides gras (biodiesel) pour la synthèse de synthons lipidiques di-fonctionnels de type polyols et polycarbonates, comme précurseurs de polyuréthanes et polyesters.

\section{Les diols}

Les premiers travaux ont porté sur la synthèse d'esters de butanediol issus d'huile de tournesol oléique, en orientant nos recherches sur une optimisation des procédés de synthèse. Les fonctions hydroxyles secondaires sont introduites par réactions successives d'époxydation et d'ouverture des cycles oxirane selon un procédé développé à grande échelle (Brevet WO2011030076; Boyer et al., 2010) (figure 14).

Dans l'optique d'accroître la réactivité de ces synthons, toute une série de polyesters polyols, de structure moléculaire variée et porteurs de fonctions hydroxyle primaires par réaction thiol-ène a ensuite été réalisée avec succès (Brevet W02011045536). Quelques structures 


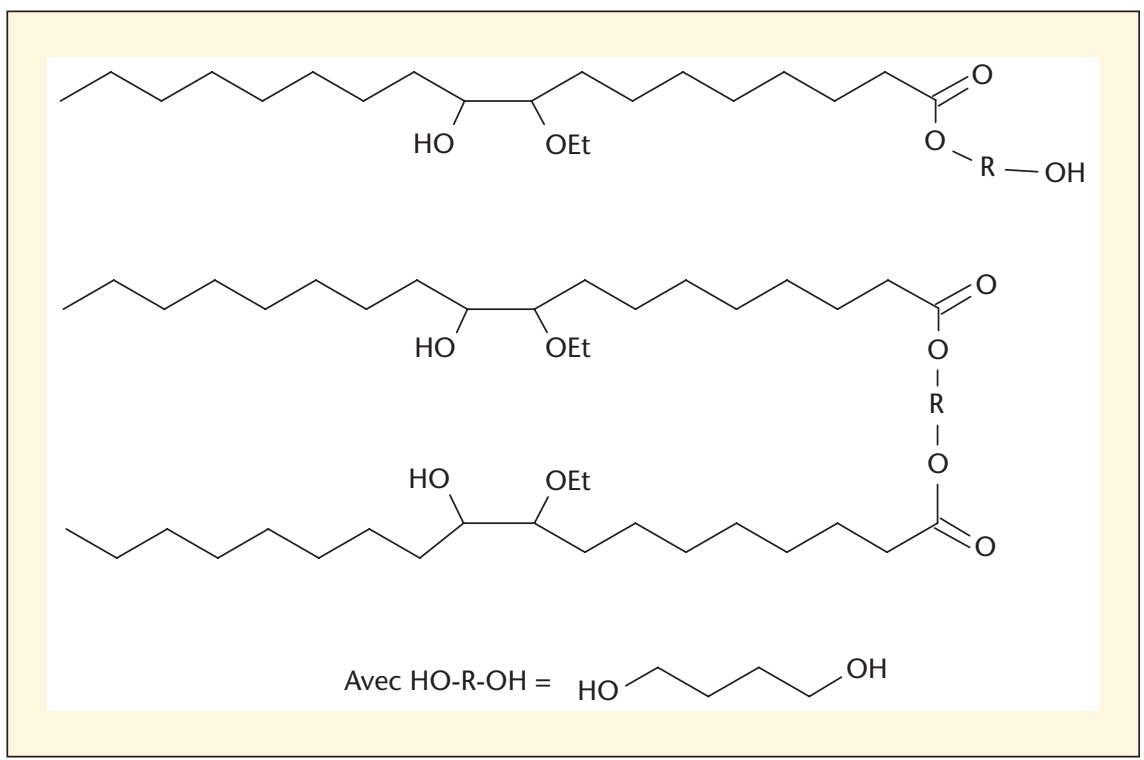

Figure 14. Exemple de polyols biosourcés obtenus après ouverture des époxydes.

chimiques sont indiquées dans la figure 15.

Une autre série de diols non ramifiés ont également été synthétisés à partir de l'oléate de méthyle et de l'undécénoate de méthyle. La transestérification ou l'aminolyse de ces esters gras avec des diols, aminoalcools ou diamines également biosourcés, conduit à des composés présentant deux insaturations terminales qui sont ensuite converties en fonctions hydroxyle par réaction thiol-ène avec le mercaptoéthanol (Brevet EP11306491).

En faisant varier la structure linéaire ou cyclique du diol employé (1,3 propane diol et isosorbide respectivement) dans l'étape de transesterification ou celle de l'amine dans l'étape d'aminolyse, il est
Synthèse des polyuréthanes et polyesters

En fonction des synthons utilisés, les polymères présentent un large panel de propriétés mécaniques et physico-chimiques. En effet, selon la fonctionnalité (nombre et nature des fonctions réactives) et la structure moléculaire de ces synthons (ou pré-polymères), des matériaux polymères linéaires thermoplastiques amorphes ou semi-cristallins ainsi que des systèmes réticulés tridimensionnels de type thermo-durs (réseaux denses) ou élastomères (réseaux lâches) peuvent être obtenus. Les premiers travaux que nous avons réalisés concernent principalement la synthèse de polyuréthanes et polyesters linéaires thermoplastiques.

\section{Les polyuréthanes}

Les polyuréthanes sont classiquement issus de la polyaddition entre un diisocyanate et un diol dans le cas de la synthèse d'un PU linéaire (thermoplastique) (figure 18). Ils sont parmi les polymères les plus polyvalents, car la gamme de monomères, en particulier les diols et les polyols, qui peuvent être utilisés dans leur synthèse, est très vaste. Notons que la part des polyols verts dans les polyuréthanes est estimée aujourd'hui à $1 \%$, d'où l'enjeu mais aussi l'intérêt que représente ce marché. La Malaisie fait partie des pays qui remplacent les polyols d'origine pétrochimique par des polyols provenant de leurs agro-ressources (en l'occurrence l'huile de palme) pour la chimie des polyuréthanes ${ }^{2}$. Ces polyols sont commercialisés par des compagnies telles que Maskimi Polyol, Green Polyol System, Bina Puri Holdings, pour une production totale de 3000 tonnes par an.

Tous les polyols synthétisés au sein de I'UMT ont été évalués pour la synthèse de nouveaux polyuréthanes et polyesters. À titre d'exemples, quelques caractéristiques des PU élaborés à partir des diols non ramifiés contenant des liens amide (FadA-diol) sont rassemblées dans le tableau 1.

Ces données montrent très clairement que l'introduction de liens amide dans le monomère diol de départ apporte une grande cohésion aux polyuréthanes ainsi

$$
\text { Avec } \mathrm{HO}-\mathrm{R}-\mathrm{OH}=\mathrm{HO} \sim \mathrm{OH} \mathrm{HO}
$$

Figure 15. Polyesters polyols biosourcés obtenus après réaction thiol-ène.
${ }^{2}$ La production d'huile de palme est estimée à 12 millions de tonnes. 
FAdE-diol 1

FAdE-diol 2<smiles>CCC(C)(C)CC(=O)O[C@H]1COC2C1OC[C@H]2OC(=O)CC(C)(C)CSCCO</smiles>

FAmE-diol 1<smiles>CC(C)(C)CC(C)(C)CC(=O)OCCCO</smiles>

FAmE-diol 2<smiles>CC(C)(C)CC(C)(C)CC(=O)O[C@H]1COC2[C@H]1OC[C@H]2O</smiles>

FAEA-diol<smiles>CC(C)(C)CC(C)(C)CC(=O)NCCCOC(=O)CC(C)(C)CC(C)(C)CSCCO</smiles>

FAmA-diol<smiles>CCC(C)(C)CC(=O)NCCCO</smiles>

FAdA-diol<smiles>CC(C)(C)CC(C)(C)CC(=O)NCCCCNC(=O)CC(C)(C)CC(C)(C)CSCCO</smiles>

Figure 16. Polyesters, polyamides et polyesteramides diols.

$$
\text { Cat. d'Hoveyda }
$$

Figure 17. Réaction de métathèse sur l'oléate de méthyle suivie des réactions de transestérification, époxydation et carbonatation. obtenus du fait de l'occurrence de liaisons hydrogène intermoléculaires. Non seulement les PU obtenus présentent des températures de transition vitreuse, $\mathrm{T}_{\mathrm{g}}$, plus élevées $\left(\mathrm{Tg}=40 / 50{ }^{\circ} \mathrm{C}\right.$ ) que celles des PU issus des diols ramifiés $\left(-40^{\circ} \mathrm{C}\right.$ à $+10^{\circ} \mathrm{C}$ ) mais ils présentent également une phase cristalline avec des températures de fusion pouvant atteindre $180^{\circ} \mathrm{C}$ dans le cas de l'emploi de diisocyanate symétrique tel le diphénylméthylène diisocyante (MDI). II est également importer de noter que les masses molaires des PU n'ont pas toujours pu être déterminées en raison de I'insolubilité des PU dans les solvants d'analyse.

Si le polyol est issu d'huiles végétales, l'isocyanate, dérivé du phosgène, est d'origine pétrochimique et présente une toxicité non négligeable. Trouver des voies alternatives de synthèse des PU sans utiliser d'isocyanate est aujourd'hui un véritable enjeu. Une façon de contourner I'utilisation des isocyanates consiste à faire réagir une di-amine sur un biscarbonate donnant l'accès à des poly (hydroxy-uréthane)s, comme indiqué dans la figure 19. Cette réaction présente aussi I'avantage de ne pas nécessiter de catalyseur.

Plusieurs poly(hydroxyuréthane)s ont été synthétisés. Des études cinétiques de la polyaddition entre diamines et biscarbonates ont été réalisées par spectroscopie ATR-IR, en suivant la disparition des bandes carbonates à 1803 et $772 \mathrm{~cm}^{-1}$ et l'apparition de bandes à $3330,1714,1530 \mathrm{~cm}^{-1}$, correspondant respectivement aux vibrations de la fonction hydroxyle, du groupement carbonyle et à la déformation de la liaison $\mathrm{N}-\mathrm{H}$ de la fonction uréthane (figure 20).

Ces études cinétiques ont permis, entre autres, de démontrer que les biscarbonates terminaux (TCFAD) sont plus réactifs que ceux intracaténaires (ICFAD). Les masses molaires des poly (hydroxyuréthane)s ainsi obtenus sont modérées et la formation de liens amide par réaction secondaire de l'amine sur les fonctions ester du monomère a été observée, notamment quand l'amine est très réactive (EDA). Des travaux sont actuellement en cours pour optimiser cette voie d'accès aux PU (tableau 2).

$D^{\prime}$ autres travaux ont également été réalisés dans le cadre de I'UMT pour trouver de nouvelles voies d'accès aux 
Tableau 1. Propriétés des polyuréthanes synthétisés à partir de diols gras contenant des fonctions amide.

\begin{tabular}{|c|c|c|c|c|c|c|c|}
\hline Polyol & Diisocyanate & $\begin{array}{l}\text { Mn } \\
(\mathrm{g} / \mathrm{mol})^{a}\end{array}$ & $\begin{array}{l}\text { Mw } \\
(\mathrm{g} / \mathrm{mol})^{\mathrm{a}}\end{array}$ & $D^{a}$ & $\begin{array}{l}\text { Tg } \\
\left({ }^{(} \mathbf{C}\right)^{b}\end{array}$ & $\operatorname{Tm}\left({ }^{C}\right)^{b}$ & $\begin{array}{l}\text { Tmc } \\
\left({ }^{\circ}\right)^{b}\end{array}$ \\
\hline \multirow{3}{*}{ FAdA-diol } & IPDI & $\begin{array}{l}20000 \\
\text { (THF) }\end{array}$ & $\begin{array}{l}30000 \\
\text { (THF) }\end{array}$ & $\begin{array}{l}1,5 \\
\text { (THF) }\end{array}$ & 40,1 & 120 & - \\
\hline & IPDI & ns & ns & ns & 47,2 & 117,3 & - \\
\hline & MDi & ns & ns & ns & 47 & $\begin{array}{l}155 / 172 / \\
178 / 173\end{array}$ & 158 \\
\hline
\end{tabular}

(a) GPC dans le DMF excepté contre-indication avec une calibration PS (b) Dsc, en mode classique de $40^{\circ} \mathrm{C}$ à $200^{\circ} \mathrm{C}, 10^{\circ} \mathrm{C} / \mathrm{min}^{\circ}$ et en mode modilé, $2^{\circ} \mathrm{C} / \mathrm{min}$

$\mathrm{HOH}_{2} \mathrm{C}-\mathrm{CH}_{2} \mathrm{OH}+\mathrm{OCN} \longrightarrow \mathrm{NCO} \longrightarrow-\mathrm{O}-\mathrm{H}_{2} \mathrm{C}-\longrightarrow-\mathrm{H}_{2} \mathrm{C}-\mathrm{O}-{ }_{\mathrm{O}} \mathrm{NH}-\longrightarrow$

Figure 18. Formation d'un polyuréthane.

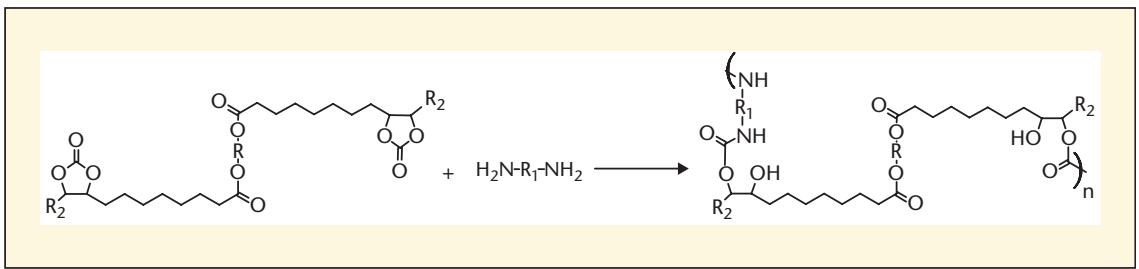

Figure 19. Formation d'un polyhydroxyuréthane par addition d'une di-amine sur un biscarbonate.

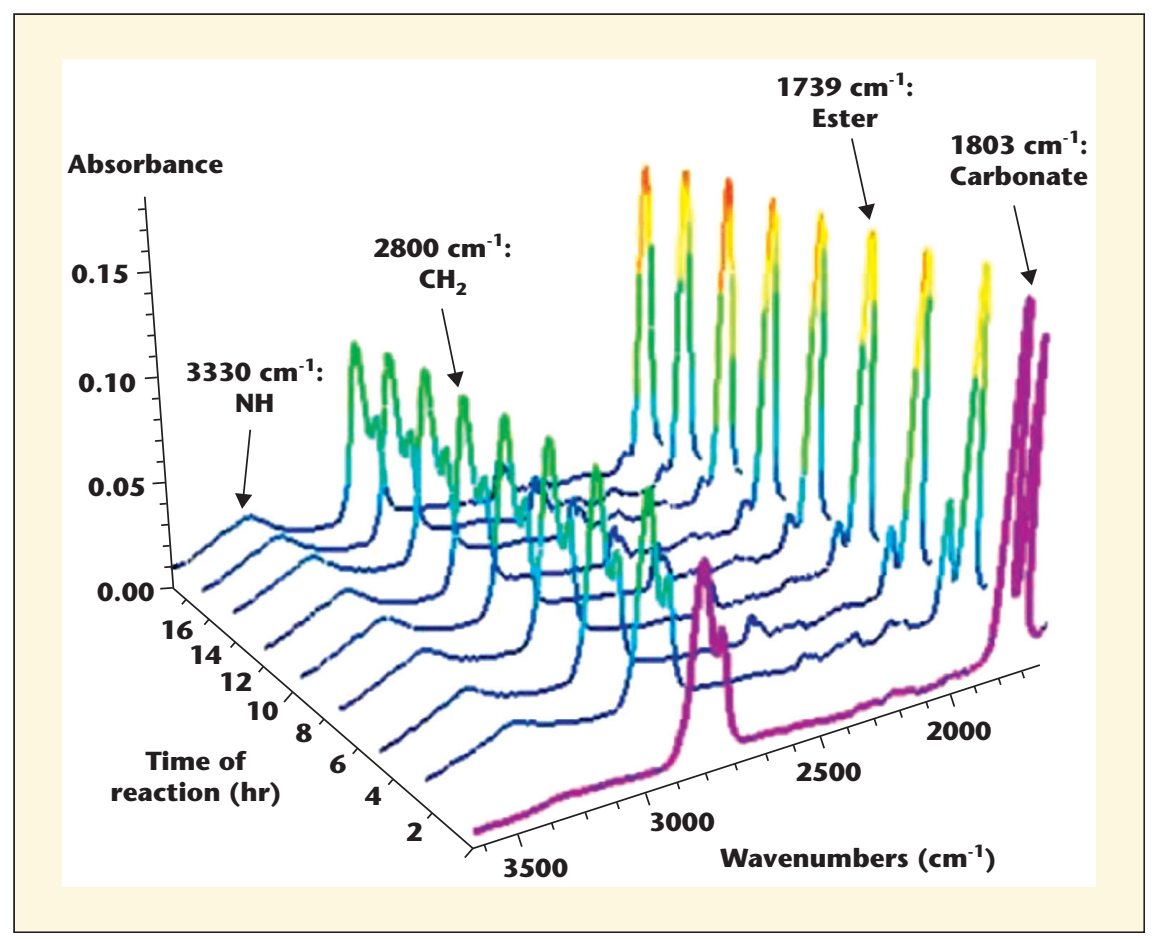

Figure 20. Contrôle par spectroscopie ATR-IR de la polyaddition à $70^{\circ} \mathrm{C}$.
PUs, évitant l'emploi de di-isocyanates, II est en effet possible d'obtenir des polyuréthanes biosourcés par auto-condensation de synthons bio-sourcés $\alpha$-hydroxy, ळ-acyl-azoture (Brevet WO2011045546 ; Palaskar et al., 2010), comme il est illustré dans la figure 21.

\section{Les polyesters}

Tous les polyols (diols) décrits précédemment ont également été testés en chimie des polyesters par réaction de condensation avec d'autres synthons diesters (ou diacides) issus de lipides, comme ceux indiqués figure 22.

Une grande variété de polyesters et poly (ester-amide)s ont été synthétisés par polycondensation de diols (figure 16) avec les diesters (figure 22) en présence du 1,5,7-triazabicyclo[4-4.0]dec-5-ène (TBD), comme catalyseur.

Les résultats obtenus sont présentés dans le tableau 3.

Comme attendu, les résultats de polymérisation ont permis de souligner des différences significatives en termes de propriétés thermomécaniques des polyesters obtenus selon la structure chimique du diol utilisé. On peut notamment observer que l'incorporation d'une unité isosorbide a pour effet de limiter la cristallinité (enthalpie de fusion plus faible) comparé aux polyesters synthétisés à partir des diols issus du propanediol. Cependant l'isosorbide a pour effet d'augmenter légèrement la température de transition vitreuse des polymères. Les polymères issus du diester $\mathrm{dE} 3$ ont un caractère semicristallin beaucoup moins prononcé en raison de la présence d'une chaîne pendante qui limite la cristallisation. Tous les matériaux issus des diesters diols et mono-esters diols présentent des $\mathrm{Tg}$ inférieures à $0^{\circ} \mathrm{C}$ et des températures de fusion relativement 
Tableau 2. Poly(hydroxyuréthane)s à partir de bis-carbonates biosourcés.

\begin{tabular}{|lllllll|}
\hline Monometres & $\mathbf{T}\left({ }^{\circ} \mathbf{C}\right)$ & $\begin{array}{l}\text { Conversion } \\
\text { totale }^{\mathbf{a}}\end{array}$ & $\begin{array}{l}\mathbf{M w} / \mathbf{g} \\
\mathbf{m o l}^{-1}\end{array}$ & $\mathbf{M w} / \mathbf{M n}$ & \% Amide & Tg $\left({ }^{\circ} \mathbf{C}\right)$ \\
\hline ICFAD/EDA & 70 & $3 \mathrm{~h}$ & 4300 & 1,1 & 22 & -25 \\
ICFAD/IPDA & 70 & $20 \mathrm{~h}$ & 9100 & 1,3 & -21 \\
ICFAD/IPDA & 110 & $12 \mathrm{~h}$ & 11700 & 1,4 & -19 \\
TCFAD/IPDA & 70 & $12 \mathrm{~h}$ & 10700 & 1,4 & -16 \\
TCFAD/IPDA & 110 & $9 \mathrm{~h}$ & 13500 & 1,5 & -13 \\
\hline
\end{tabular}

(a) à disparition complète de la bande à $772 \mathrm{~cm}-1$. (b) DSC, rampe de température de $-100{ }^{\circ} \mathrm{C}$ to $200{ }^{\circ} \mathrm{C}$ à $10^{\circ} \mathrm{Cmin}-1$.

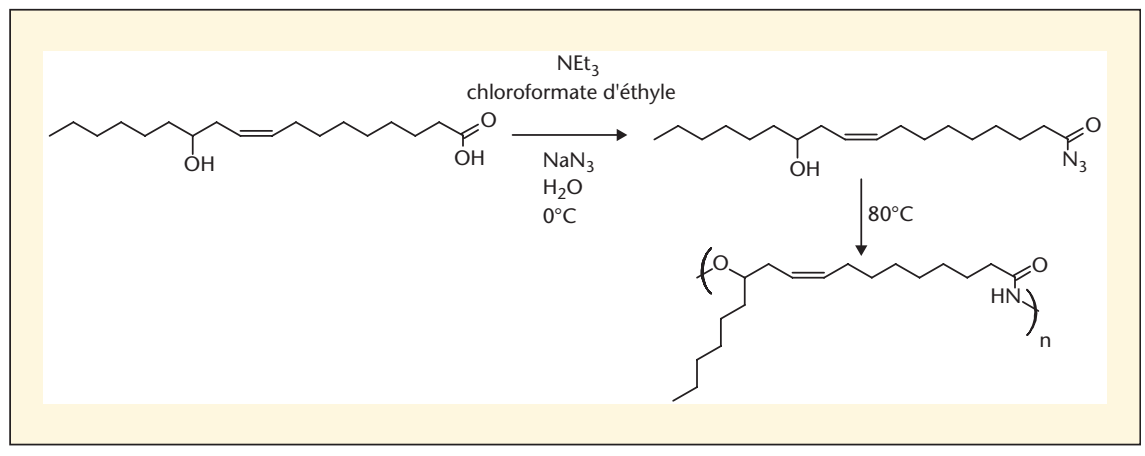

Figure 21. Formation d'un polyuréthane par auto-condensation. faibles (entre 0 et $40^{\circ} \mathrm{C}$ ). Ces propriétés sont donc insuffisantes pour un grand nombre d'applications.

Pour pallier cet inconvénient, le FAdAdiol contenant des liens amide a été synthétisé et utilisé en substitution des diesters diols. Les polyesters ainsi obtenus présentent une température de fusion voisine de $120^{\circ} \mathrm{C}$ et des températures de transition vitreuse autour de $0{ }^{\circ} \mathrm{C}$, ce qui rend ces polyesters très compétitifs en regard d'autres polyesters aliphatiques.

Tableau 3. Caractérisations des polyesters issus de la polycondensation entre les diols (figure 8) et les diesters (figure 22).

\begin{tabular}{|c|c|c|c|c|c|c|c|c|}
\hline Diol & Diester & $\begin{array}{l}\text { Mw } \\
(\mathrm{g} / \mathrm{mol})^{a}\end{array}$ & $\mathbf{M w} / \mathbf{M n}^{\mathbf{a}}$ & $\operatorname{Tg}\left({ }^{\circ} \mathrm{C}\right)^{\mathbf{b}}$ & $\operatorname{Tm}_{1}\left({ }^{\circ} \mathbf{C}\right)^{\mathbf{b}}$ & $\Delta H m_{1}(J / g)^{b}$ & $\operatorname{Tm}_{2}\left({ }^{\circ}\right)^{b}$ & $\Delta \mathrm{Hm}_{2}(\mathbf{J} / \mathbf{g})^{\mathbf{b}}$ \\
\hline \multirow[t]{4}{*}{ FAdE-diol 1} & $\mathrm{dE} 1$ & 35041 & 1,9 & $-49,4$ & 36,1 & 79,9 & - & - \\
\hline & $\mathrm{dE} 2$ & 33293 & 1,5 & $-39,3$ & 38,7 & 74,1 & - & - \\
\hline & $\mathrm{dE} 3$ & 15862 & 1,9 & $-58,1$ & 9,1 & 7,2 & 22,6 & 20,5 \\
\hline & dE4 & 34547 & 1,6 & $-46,6$ & 45,1 & 76,6 & - & - \\
\hline \multirow[t]{4}{*}{ FAdE-diol 2} & $\mathrm{dE1}$ & 13318 & 1,5 & $-40,1$ & 29,9 & 31,4 & 37,2 & 2,4 \\
\hline & $\mathrm{dE} 2$ & 17410 & 1,5 & $-30,9$ & 36,7 & 45,2 & - & - \\
\hline & $\mathrm{dE3}$ & 22984 & 2,3 & $-46,8$ & 12,9 & 4,8 & 23,7 & 14,7 \\
\hline & dE4 & 16387 & 1,5 & -31.9 & 35,1 & 47,9 & - & - \\
\hline \multirow[t]{4}{*}{ FAmE-diol 1} & $\mathrm{dE} 1$ & 7865 & 1,3 & $-27,4$ & 32,3 & 24,6 & - & - \\
\hline & $\mathrm{dE} 2$ & 27333 & 2,1 & $-38,3$ & 18 & 41,4 & 29,8 & 51,8 \\
\hline & $\mathrm{dE} 3$ & 33050 & 1,6 & $-61,4$ & $-5,9$ & 17,8 & - & - \\
\hline & $\mathrm{dE} 4$ & 7037 & 1,2 & $-44,8$ & 37,4 & 76,5 & - & - \\
\hline \multirow[t]{4}{*}{ FAmE-diol 2} & $\mathrm{dE1}$ & 14992 & 1,5 & $-39,9$ & 15,5 & 6,5 & 25,4 & 6,9 \\
\hline & $\mathrm{dE} 2$ & 18374 & 1,2 & $-28,5$ & 16,4 & 40,8 & 25,6 & 2,7 \\
\hline & $\mathrm{dE3}$ & 33744 & 1., & $-43,8$ & $-5,2$ & 7,7 & 10,5 & 0,7 \\
\hline & dE4 & 21034 & 1,2 & $-30,5$ & 21 & 31 & - & - \\
\hline \multirow[t]{4}{*}{ FAdA-diol } & $\mathrm{dE} 1$ & 29028 & 1,5 & -10 & 121,1 & 64,3 & 126,5 & 7,1 \\
\hline & $\mathrm{dE} 2$ & 12641 & 1,3 & 2 & 119,8 & 31,3 & 125,7 & 15,8 \\
\hline & $\mathrm{dE} 3$ & 17610 & 1,6 & -13 & 118,3 & 63,83 & - & - \\
\hline & dE4 & 9524 & 1,5 & 5 & 129,3 & 8,7 & 138,4 & 32,64 \\
\hline
\end{tabular}

Catalyseur : TBD $/ 10 \%$ par fonction ester (a) SEC THF clibration PS (b) DSC, $-80^{\circ} \mathrm{C}$ à $170{ }^{\circ} \mathrm{C}, 10^{\circ} \mathrm{C} / \mathrm{min}(\mathrm{c}) \mathrm{TGA}, 25^{\circ} \mathrm{C}$ à $700{ }^{\circ} \mathrm{C}, 10^{\circ} \mathrm{C} / \mathrm{min}$ 


$$
\text { (c) }
$$

dE4

Figure 22. Structure des diesters issus des lipides pour la synthèse de polyesters.

\section{Conclusion}

Parmi les grands enjeux actuels de la recherche en chimie, la valorisation de la biomasse pour la production d'intermédiaires chimiques bien définis reste un véritable défi scientifique, technologique et environnemental. Les recherches menées au sein de I'UMT Polygreen s'inscrivent dans cette démarche et visent la mise au point de nouveaux synthons lipidiques pour la production de polymères bio-sourcés de spécialité. Des résultats encourageants ont été obtenus en chimie des polyuréthanes et des polyesters. Ces recherches nécessitent encore de nombreux approfondissements et optimisations notamment pour rendre les procédés de synthèse et production plus efficients et performants, condition nécessaire à la substitution (même partielle) des dérivés de la pétrochimie par ceux issus de la biomasse.

L'ITERG et sa plateforme technologique CEDOP (Centre Européen de Développement des Oléo-Produits) sont en parfaite adéquation avec ces objectifs et répondent parfaitement aux attentes en termes d'innovation et de développement de la chimie verte.

\section{RÉFÉRENCES}

Bantchev GB, Kenar JA, Biresaw G, Han MG. Free radical addition of butanethiol to vegetable oil double bonds. I Agric Food Chem 2009 ; 57 : 1282-90.

Boyer A, Cloutet E, Tassaing T, Gadenne B, Alfos $\mathrm{C}$, Cramail $\mathrm{H}$. Solubility in $\mathrm{CO} 2$ and carbonation studies of epoxidized fatty acid diesters : towards novel precursors for polyurethane synthesis. Green Chem 2010 ; 12 : 2205-13.

Brevet W02011061452, inventeurs : Cramail H, Boyer A, Cloutet E, Gadenne B, Alfos C. Précurseurs bis-carbonates, leur procédé de préparation et leurs utilisations.

Brevet WO2011030076, inventeurs : Cramail H, Boyer A, Cloutet E, Bakhiyi R, Alfos C. Nouveau procédé de préparation de polyols et produits tels qu'obtenus.

Brevet W02011045536, inventeurs : Cramail H, Boyer A, Cloutet E, Alfos C. Nouveau procédé de préparation de polyols par thiolisation et produits tels qu'obtenus.

Brevet WO2011045546, inventeurs: Cramail H, Boyer A, Palaskar D, Cloutet E, Alfos
C. Synthèse de polyuréthanes à partir d'huile de tournesol et d'huile de ricin par autocondensation.

Brevet EP11306491, inventeurs : Cramail H, Maisonneuve L, Lebarbé T, Cloutet E, Gadenne B, Alfos C. New process of preparation of polyols and polyamines, and products as obtained.

De Torres M, Jiménez-Osés G, Mayoral JA, Pires E. Fatty acid derivatives and their use as CFPP additives in biodiesel. Bioresour Technol $2011 ; 102$ : 2590-4.

Foltran S, Maisonneuve L, Cloutet $\mathrm{E}$, et al. Solubility in $\mathrm{CO}_{2}$ and Swelling studies by in situ IR spectroscopy of Vegetable-Based Epoxidized Oils as Polyurethane precursors. Polym Chem 2012; DOI: 10.1039/ C2PY00476C.

Galia M, Montero de Espinosa L, Ronda JC, Lligadas G, Cadiz V. Vegetable oil-based thermosetting polymers. Eur / Lipid Sci Technol 2010 ; 112 : 87-96.

Güner FS, Yagci Y, Erciyes AT. Polymers from triglyceride oils. Prog Polym Sci 2006 ; 31 : 633-70.

Meher LC, Vidya Sagar D, Naik SN. Technical aspects of biodiesel production by transesterification. Renew Sustain Energy Rev 2006 ; $10: 248-68$.

Nakatani N, Takamori H, Takeda K, Sakugawa $\mathrm{H}$. Transesterification of soybean oil using combusted oyster shell waste as a catalyst. Bioresour Technol 2009; 100: 1510-3.

Narine S, Yue J, Kong X. Production of Polyols from Canola Oil and their Chemical Identification and Physical Properties. I Am Oil Chem Soc 2007; 84 : 173-9.

Palaskar D, Boyer A, Cloutet E, Alfos C, Cramail H. Synthesis of Biobased Polyurethane from Oleic and Ricinoleic Acids as the Renewable Resources via the AB-Type SelfCondensation Approach. Biomacromolecules 2010 ; 11 : 1202-11.

Palaskar D, Boyer A, Cloutet E, et al. Original Diols from Sunflower and Ricin Oils: Synthesis, Characterization and Use as Polyurethane Building Blocks. Journal of Polymer Science, Part A, Polym Chem 2012 (accepted).

Petrovic ZS, Cevallos MJ, Javni I, Schaefer DW, Justice R. Soy-oil-based segmented polyurethanes. J Polym Sci Part B, Polym Phys $2005 ; 43$ : 3178-90.

Petrovic ZS, Cretkovic I, Hong DP, et al. Vegetable oil-based triols from hydroformylated fatty acids and polyurethane elastomers. Eur J Lipid Sci Technol 2010; 112: 97-102.

Petrovic ZS, Guo A, Zhang W. Structure and properties of polyurethanes based on halo- 
genated and nonhalogenated soy-polyols. J Polym Sci, Part A, Polym Chem 2000 ; 38 : 4062.

Petrovic ZS, Zhang W, Javni I. Structure and Properties of Polyurethanes Prepared from Triglyceride Polyols by Ozonolysis. Biomacromolecules 2005 ; 6 : 713-9.

Rapport ADEME 2011 : Usage des résines biosourcées: quels développements en
France, dans I'Union Européenne et dans le monde?.

Rapport PIPAME 2010 : Mutations économiques dans le domaine de la chimie.

Sharma V, Kundu PP. Addition polymers from natural oils - A review. Prog Polym Sci 2006 ; 31 : 983-1008.

Zlatanic A, Lava C, Zhang W, Petrovic ZS. Effect of structure on properties of polyols and polyurethanes based on different vegetable oils. I Polym Sci, Part B, Polym Phys $2004 ; 42$ : 809-819.

Zlatanic A, Petrovic ZS. J Polym Mater 2009 : 103-29.

Zlatanic A, Petrovic ZS, Dusek K. Structure and Properties of Triolein-Based Polyurethane Networks. Biomacromolecules 2002 ; 3 : 1048-56. 